\author{
С.С. Алешкина ${ }^{1}$, М.Е. Лихачев ${ }^{1}$, Д.С. Липатов ${ }^{2,3}$, \\ О.И. Медведков ${ }^{1}$, К.К. Бобков ${ }^{1}$, М.М. Бубнов ${ }^{1}$, А.Н. Гурьянов ${ }^{2}$ \\ ${ }^{1}$ Научный центр волоконной оптики РАН, Москва, Россия \\ ${ }^{2}$ Институт химии высокочистых веществ им. Г.Г. Девятых РАН, \\ Нижний Новгород, Россия \\ ${ }^{3}$ Нижегородский государственный университет им. Н.И. Лобачевского, \\ Нижний Новгород, Россия

\section{Полностью волоконный источник МОЩНОГО ЛАЗЕРНОГО ИЗЛУЧЕНИЯ С ДЛИНОЙ ВОЛНЫ ГЕНЕРАЦИИ 0,98 МКМ}

\begin{abstract}
Реализована конструкция непрерывного одномодового полностью волоконного лазера с длиной волны генерации 977 нм и выходной мощностью 5,5 Вт. Ключевым элементом лазерной схемы являлся иттербиевый волоконный световод со ступенчатым профилем показателя преломления, с увеличенным диаметром одномодовой сердцевины (30 мкм). Снижение внешнего диаметра оболочки позволило увеличить поглощение из оболочки: отношение эффективного диаметра оболочки к диаметру сердцевины составляло 1:3,4, что обусловило высокую эффективность лазера (25\%).
\end{abstract}

Ключевые слова: волоконный лазер, иттербий, световод с большой площадью поля моды.

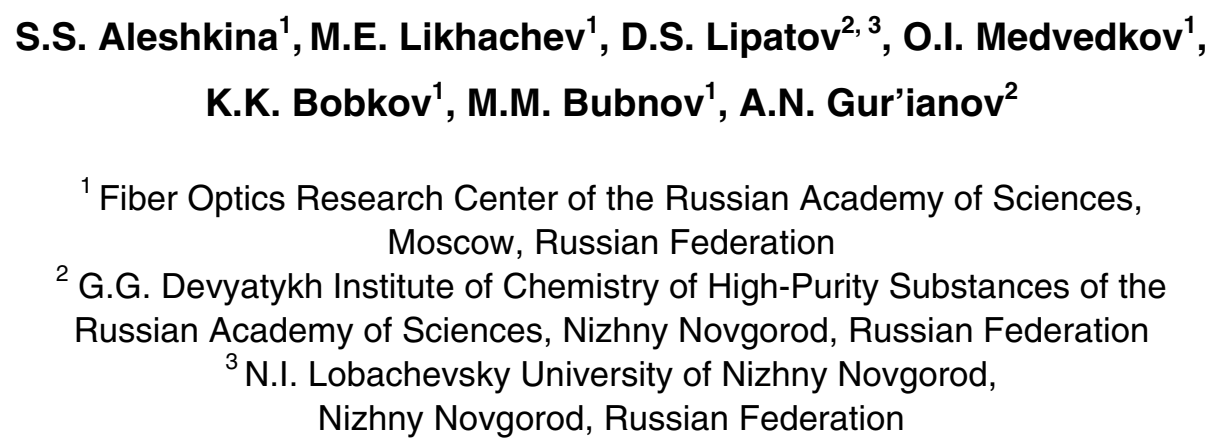

\title{
HIGH POWER ALL-FIBER LASER AT 0,98 UM
}

All-fiber single-mode continuous-wave laser with output power as high as 5,5 W at wavelength of $977 \mathrm{~nm}$ was realized. A key element of the laser scheme was Yb-doped step-index fiber with an increased single-mode core diameter of 30 microns. Reducing of the cladding diameter allowed us to increase the absorption from the cladding (ratio of diameters of the core and cladding was $1: 3,4$ ) and laser efficiency (25\%), correspondingly.

Keywords: fiber laser, ytterbium, large mode area fiber. 
Одномодовые лазеры с длиной волны генерации около 0,98 мкм нашли широкое применение в спектроскопии, а также в качестве источников накачки для эрбиевых и иттербиевых волоконных систем. Удвоение частоты такого лазера позволяет использовать его вместо массивного и дорогостоящего лазера на аргоне. Однако коммерчески доступными на данный момент одномодовыми источниками на длине волны 0,98 мкм являются только полупроводниковые пигтелированные волоконным световодом диоды, выходная мощность которых ограничена величиной 1 Вт.

Среди возможных способов создания более мощных источников лазерного излучения стоит выделить реализацию лазеров на волоконных световодах, легированных ионами иттербия. Ионы иттербия обладают широкой полосой люминесценции - от 920 до 1150 нм. При этом возможность лазерной генерации была продемонстрирована для спектрального диапазона 970-1150 нм. Стоит отметить, что сложность создания лазеров, работающих в спектральном диапазоне 970-980 нм, связана с тем, что в этом случае излучательные переходы осуществляются по квазитрехуровневой схеме, в отличие от переходов, происходящих на длинах волн 1010-1150 нм, где лазер работает по квазичетырехуровневой схеме. Как следствие, основной проблемой при создании лазера, работающего вблизи длины волны 976 нм, является высокий порог генерации, связанный с необходимостью создания инверсии более величины 50\% по всей длине световода и конкуренция с генерацией по четырехуровневой схеме (на длине волны 1030 нм). В данном случае наиболее критическим является достижение высокой плотности мощности накачки в сердцевине (как правило, ее отношение к плотности мощности сигнала должно быть выше 0,2-0,5 в зависимости от используемой схемы лазера или усилителя). С этой точки зрения наиболее простым способом реализации мощного источника на 0,98 мкм является использование схемы с накачкой по сердцевине [1-3]. В то же время выходная мощность доступных одномодовых источников на 910-940 нм, выступающих в качестве источников накачки, ограничена мощностью в несколько ватт. Вследствие этого наилучшие результаты по генерации одномодового лазерного излучения на 0,98 мкм в схеме с накачкой по сердцевине не превышают 2,1 Вт излучения [1]. Более того, реализация источников накачки, работающих в области 910-940 нм (например, лазер на неодимовом волоконном световоде [1, 3]), является отдельной, достаточно сложной с практической точки зрения задачей. 
Гораздо более перспективным подходом в плане увеличения выходной мощности одномодовых лазеров на 0,98 мкм является использование схем с накачкой по оболочке [4-9]. В то же время стандартные волоконные световоды с накачкой по оболочке, как правило, имеют площадь первой оболочки на два порядка больше, чем площадь сердцевины. Как следствие, для достижения инверсии более 50 \% полная мощность накачки должна превышать мощность генерируемого сигнала больше, чем на порядок, что приводит к ничтожно малой эффективности таких лазеров (во избежание появления генерации на длине волны 1030 лазер приходится делать коротким, что приводит к большой доле непоглощенной накачки). Повышение эффективности генерации на длине волны 976 нм может быть достигнуто путем увеличения отношения площади сердцевины и первой оболочки [10]. Для этих целей необходимо использовать световоды с большой площадью поля моды и малым размером внутренней отражающей оболочки. При этом в случае световодов со ступенчатым профилем показателя преломления увеличение размера сердцевины световода и сохранение одномодового режима распространения требует уменьшения показателя преломления сердцевины до уровня, близкого к уровню нелегированного кварцевого стекла, из которого изготовлена отражающая оболочка. Более того, увеличивающаяся чувствительность таких световодов к изгибу приводит к необходимости использования достаточно специфических типов световедущих структур.

Так, наилучшие результаты (94 Вт на длине волны 977 нм) были получены на негнущемся (диаметр внешней оболочки имеет миллиметровую размерность) микроструктурированном световоде-стержне, волноводные свойства которого обусловлены наличием в оболочке тонких воздушных капилляров (Photonic crystal Fiber) [9] (соотношение диаметров сердцевины и внутренней оболочки составило 6,25:1). Второй по выходной мощности результат (10 Вт оптического излучения на 976 нм) принадлежит схеме, в которой в качестве активного элемента использован иттербиевый световод со ступенчатым профилем показателя преломления и изменяющимися по длине поперечными размерами (соотношение диаметров сердцевины и внутренней оболочки составило 8,5:1) [6]. Тонкий конец световода был адаптирован для сварки со световодами стандартного типа, в то время как толстый конец световода имел диаметр 1 мм. В работе [8] 4,3 Вт излучения на 977 нм было 
получено на световоде с воздушной оболочкой в схеме усилителя с накачкой навстречу (диаметр внутренней оболочки составил 25 мкм).

Главным недостатком предложенных конструкций специальных световодов являлась невозможность создания полностью волоконных схем лазера: использование предложенных конструкций световодов в схемах требовало привлечения объемных элементов (систем ввода/вывода излучения в световод/из световода $[6,8,9]$, дихроичных зеркал [8, 9], зеркал, образующих резонатор [9]). При этом нарушение монолитности лазерной системы приводит к ухудшению надежности, чувствительности, к изменениям окружающей среды, громоздкости и, более того, сложности дальнейшего использования полученного излучения в волоконных схемах.

Целью настоящей работы являлась реализация конструкции технологически простого в изготовлении волоконного световода, адаптированного для осуществления эффективной генерации на длине волны около 0,98 мкм и реализация на его основе полностью волоконной лазерной схемы.

\section{1. Реализация активного волоконного световода}

Стоит отметить, что помимо сложности достижения лазерной генерации в области длин волн около 976 нм не менее важным является решение проблемы фотопотемнения [11], проявляющегося в снижении эффективности генерации иттербиевого лазера во время его эксплуатации. Данный эффект обусловлен наведением дефектов в сетке стекла, имеющих широкую полосу поглощения с максимумом в видимой области спектра [12-13]. Природа дефектов связана с образованием кислородо-дырочных центров как результата фотоиндуцированного возбуждения состояния переноса заряда ионов $\mathrm{Yb}^{3+}[14-17]$. При этом основной проблемой является то, что скорость фотопотемнения пропорциональна инверсии ионов иттербия в шестой степени [12], т.е. по сравнению с лазерами, работающими в диапазоне 1060 нм (инверсия около $5 \%$ ), скорость наведения потерь за счет фотопотемнения при генерации в области 976 нм оказывается на шесть порядков выше.

Таким образом, для достижения долговременной работы волоконного лазера необходимо использовать стеклянную матрицу, не чувствительную к данному эффекту. Среди наименее чувствительных к эффекту фотопотемнения стеклянных матриц можно выделить фос- 
форосиликатную матрицу $\mathrm{P}_{2} \mathrm{O}_{5}-\mathrm{SiO}_{2}$ [12] и алюмосиликатную матрицу, дополнительно легированную ионами церия $\mathrm{Al}_{2} \mathrm{O}_{3}-\mathrm{Ce}_{2} \mathrm{O}_{3}-\mathrm{SiO}_{2}$ [18]. Однако как введение оксида фосфора, так и совместное введение оксида алюминия с оксидом церия приводит к увеличению показателя преломления сердцевины относительно уровня кварцевого стекла и, следовательно, ограничивает максимальный диаметр сердцевины одномодового световода. Эффективное введение редкоземельного элемента в сетку стекла без существенного увеличения показателя преломления сердцевины относительно уровня кварца может быть реализовано в фосфороалюмосиликатной матрице [19-21], также практически не подверженной деградации сетки стекла в результате фотопотемнения [21].

Как было указано выше, одним из основных факторов, определяющих эффективность волоконного лазера, работающего в области 976 нм, является отношение диаметров сердцевины и оболочки. С целью увеличения диаметра сердцевины при выборе световедущей структуры нами был использован световод, имеющий $\mathrm{W}$-профиль: сердцевина со ступенчатым профилем показателя преломления окружена депрессированным слоем (с показателем преломления ниже уровня кварцевого стекла). Известно, что такая конструкция обладает уменьшенной длиной волны отсечки при фиксированном диаметре и показателе преломления сердцевины по сравнению с обычными световодами со ступенчатым профилем показателем преломления (СППП). Это позволяет обеспечивать больший диаметр сердцевины при сохранении одномодового режима распространения. Не менее важно, что световоды c $\mathrm{W}$-профилем обладают меньшей чувствительностью к изгибу по сравнению с обычными СППП-световодами. Это позволяет еще более снизить показатель преломления сердцевины световода и соответственно увеличить ее диаметр.

Заготовка активного световода была изготовлена методом MCVD. Ионы иттербия вводились в сетку стекла методом газофазной пропитки [22] с использованием летучего соединения ДПМ (Yb). Стоит отметить, что малый показатель преломления сердцевины также накладывал ограничение на максимальную концентрацию оксида иттербия. Так, при показателе преломления сердцевины относительно уровня кварцевого стекла, не превышающем 0,0007, нами было введено 0,1 вес. \% оксида иттербия. С целью уменьшения перепоглощения полезного сигнала в работе была использована методика селективного легирования [11], заключающаяся в отсутствии легирования центральной области сердце- 
вины (в нашем случае 6 \% от площади сердцевины), где фундаментальная мода имеет наибольшую интенсивность.

При выборе поперечных параметров световода было учтено, что диаметр оболочки должен быть как можно меньше, но при этом должна быть сохранена возможность скалывания и сварки световода при помощи стандартного оборудования, что соответствует диаметру, равному 80 мкм. Кроме того, чтобы обеспечить эффективное перемешивание мод накачки, нами была нарушена цилиндрическая симметрия кварцевой оболочки: заготовка была сполирована до формы квадрата. В итоге отношение диаметра сердцевины к эффективному диаметру оболочки, рассчитанному по площади сечения световода, составило 1/3,4.

Световод с внешними размерами по кварцевой оболочке $80 \times 80$ мкм был вытянут в отражающем полимере, обеспечивающем распространение излучения накачки по оболочке с апертурой 0,46. Диаметр сердцевины составил около 30 мкм, что при реализованных параметрах профиля показателя преломления обеспечивало одномодовый режим распространения на длине волны 0,98 мкм. Оцененный диаметр поля моды был равен 18 мкм. Профиль показателя преломления световода и распределение интенсивности поля фундаментальной моды приведены на рис. 1. Изображение торца световода, выполненное посредством оптического микроскопа, приведено на вставке рис. 1. На рис. 2 приведен измеренный спектр поглощения по оболочке.

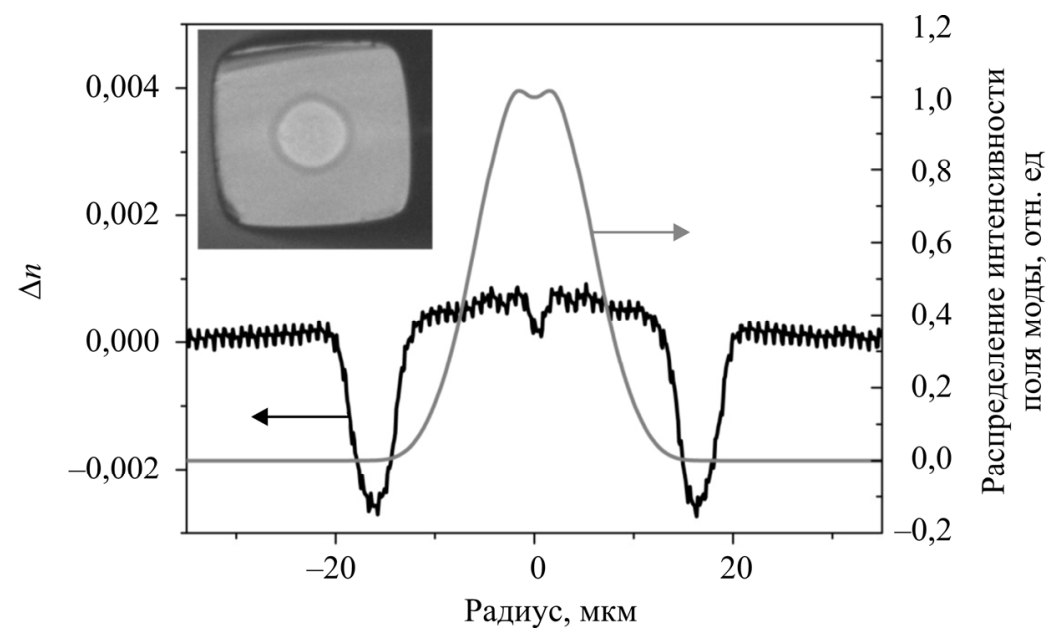

Рис. 1. Измеренный профиль показателя преломления световода, рассчитанного по профилю распределения интенсивности поля фундаментальной моды; на вставке - изображение торца световода 


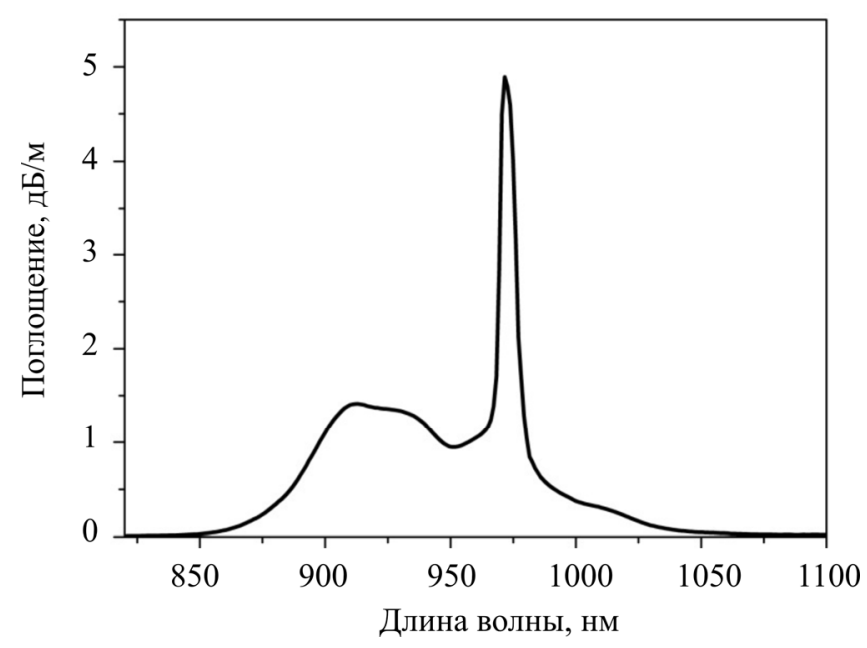

Рис. 2. Измеренное поглощение по оболочке реализованного световода

\section{2. Схема лазера и основные результаты}

Реализованный световод был протестирован в схеме непрерывного лазера $(\lambda=976,8$ нм) с накачкой по оболочке (рис. 3). Брэгговская решетка с коэффициентом отражения $100 \%$ была записана на одномодовом на рабочей длине волны световоде 20/80 мкм. Полупрозрачная решетка была записана на маломодовом на длине волны 976,8 нм световоде 20/125 мкм. Выбор коэффициента отражения полупрозрачной брэгговской решетки был обусловлен необходимостью извлечения максимума полезного сигнала [23] и составил $10 \%$. Активный световод был намотан на катушку диаметром 30 см. Длина активного световода составила около 4 м.

Излучение накачки подводилось со стороны глухой на рабочей длине волны решетки. В качестве источника накачки был использован коммерчески доступный полупроводниковый диод мощностью 33 Вт с волоконным выходом на основе световода 105/125 мкм $(\lambda=915 \pm 10$ нм, $\mathrm{NA}=0,15)$. Также в схеме для согласования световода на выходе из многомодового источника накачки и световодов, из которых изготовлен резонатор, был использован световод-конус - многомодовый кварц-полимерный световод, диаметр кварцевой оболочки которого плавно менялся от 110 до 80 мкм. Введение в схему световода-конуса позволяло избежать потери мощности накачки вследствие рассогласованности диаметров световедущих элементов системы. Вследствие со- 
хранения яркости излучения апертура распространяющегося излучения накачки увеличивалась после прохождения световода-конуса до величины порядка 0,26 .

После $10 \%$ брэгговской решетки непоглощенное излучение накачки устранялось из первой оболочки (рис. 3). Торец выходного световода был сколот под углом для устранения паразитного обратного отражения на всех длинах волн и усиления на нежелательных длинах волн соответственно.

На рис. 4 приведена измеренная зависимость выходной мощности лазера от мощности накачки на выходе из полупроводникового диода, на вставке показан спектр выходного излучения. Порог лазерной

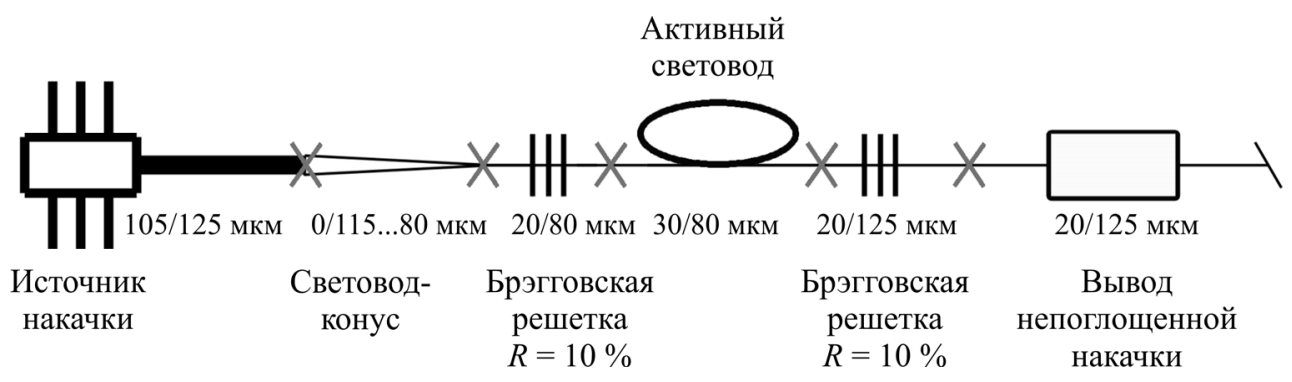

Рис. 3. Использованная схема лазера. Цифрами снизу обозначены геометрические параметры световодов: размер сердцевины/размер оболочки

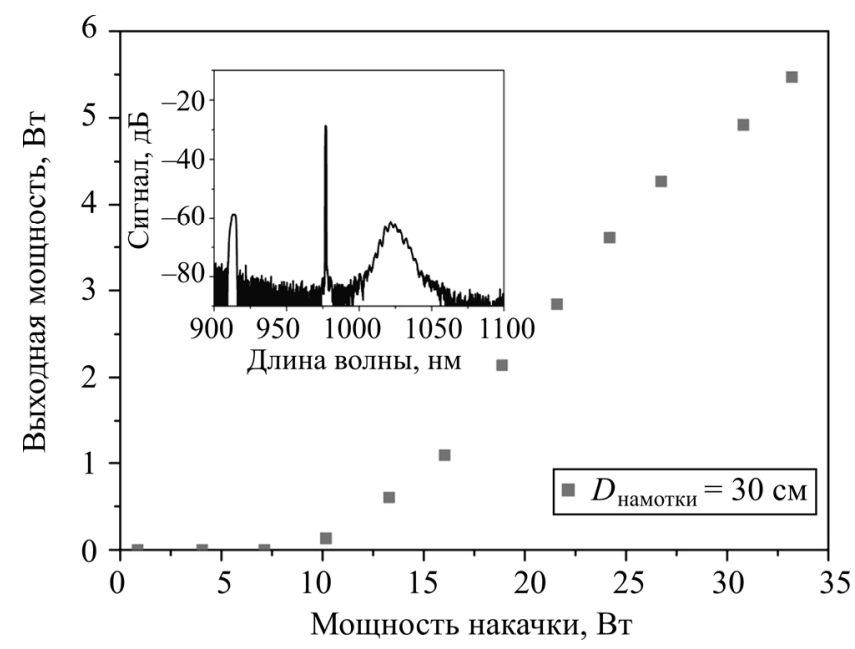

Рис. 4. Зависимость мощности выходного излучения на 976,8 нм от мощности накачки. На вставке приведен спектр выходного излучения, измеренный при максимальной мощности накачки 
генерации составил около 10 Вт. Соотношение сигнал/шум было оценено в $\approx 33$ дБ. Дифференциальная эффективность преобразования мощности накачки в сигнал составила $25 \%$.

\section{3. Заключение}

В работе реализована и протестирована в схеме непрерывного лазера конструкция иттербиевого специального волоконного световода с малым соотношением геометрических параметров оболочки и сердцевины: сторона катета кварцевой оболочки составляла 80 мкм, диаметр сердцевины - 30 мкм. Выходная мощность лазера, к выходному концу которого был приварен пассивный световод стандартного типа 20/125 мкм в выводящем полимере, составила 5,5 Вт $(\lambda=976,8$ нм). Насколько нам известно, полученный результат является рекордным для полностью волоконных одномодовых лазерных систем с длиной волны генерации около 980 нм. Кроме того, стоит отметить, что полученный в работе результат был ограничен лишь доступной мощностью накачки и может быть улучшен при использовании в схеме более мощных источников.

Авторы выражают благодарность директору Научного центра волоконной оптики РАН академику Е.М. Дианову за постоянную поддержку и проявленный интерес к настоящей работе.

Работа выполнена при частичной финансовой поддержке гранта РФФИ 15-38-20923 мол_а_вед и программы «УМНИК».

\section{Список литературы}

1. Bartolacci C., Laroche M., Gilles H. All-fiber Yb-doped CW and pulsed laser sources operating near $980 \mathrm{~nm} / /$ Advanced Solid-State Photonics, OSA Technical Digest (CD) (Optical Society of America). - 2011. Paper ATuB9.

2. $977 \mathrm{~nm}$ all fiber DFB laser / L.B. Fu, M. Ibsen, D.J. Ridcharson, D.N. Payne // IEEE Phot. Tech.Let. - 2004. - Vol. 16 - P. 2442-2445.

3. $0.65 \mathrm{~W}$ single-mode $\mathrm{Yb}$-fiber laser at $980 \mathrm{~nm}$ pumped by $1.1 \mathrm{~W}$ Nd:YAG / L.A. Zenteno, J.D. Minelly, M. Dejneka, S. Crigler // Advanced Solid State Lasers, OSA Technical Digest Series (Optical Society of America. - 2000. - Paper TuC8.

4. High-power $\mathrm{Yb}$-doped double-clad fiber lasers for a range of 0.98-1.04 $\mu$ m / A.S. Kurkov, O.I. Medvedkov, V.M. Paramonov, S.A. Va- 
siliev, E.M. Dianov, V. Solodovnikov // Optical Amplifiers and Their Applications, OSA Technical Digest Series (Optical Society of America. 2001. - Paper OWC2.

5. A 3.5 W $977 \mathrm{~nm}$ Cladding-pumped Jacketed Air-Clad YtterbiumDoped Fiber Laser / K.H. Ylä-Jarkko, R. Selvas, D.B.S. Son, J.K. Sahu, C.A. Codemard, J. Nilsson, S.A. Alam, A.B. Grudinin // Advanced SolidState Photonics. Vol. 83 of OSA Trends in Optics and Photonics (Optical Society of America). - 2003. - Paper 103.

6. Investigation of a large core $976 \mathrm{~nm} \mathrm{Yb}$ fiber laser for high brightness fiber-based pump sources / M. Leich, M. Jäger, S. Grimm, J. Dellith [et al.] // Proc. SPIE 8961, Fiber Lasers XI: Technology, Systems, and Applications, 89610Z (March 7, 2014). DOI: 10.1117/12.2039169

7. Efficient $\mathrm{Yb}$ fiber laser at $980 \mathrm{~nm}$ pumped by the high-brightness semiconductor source / A.S. Kurkov, E.M. Dianov, V.M. Paramonov, O.I. Medvedkov, S.A. Vasiliev, V. Solodovnikov, V. Zhilin, A.Y. Laptev, A.A. Umnikov, A.N. Guryanov // Conference on Lasers and Electro-Optics / OSA Technical Digest (Optical Society of America). - 2001. - Paper CTuQ2. 8. A $4.3 \mathrm{~W} 977 \mathrm{~nm}$ ytterbium-doped jacketed-air-clad fiber amplifier / D.B.S. Soh, C. Codemard, J.K. Sahu, J. Nilsson, V. Philippov, C. Alegria, Y. Jeong // Advanced Solid-State Photonics, OSA Technical Digest (Optical Society of America). - 2004. - Paper MA3.

9. High power ytterbium-doped rod-type three-level photonic crystal fiber laser / J. Boullet, Y. Zaouter, R. Desmarchelier, M. Cazaux, F. Salin, J. Saby, R. Bello-Doua, E. Cormier // Opt. Express - 2008 - Vol. 16. P. 17891-17902.

10. Ring-doped cladding-pumped single-mode three-level fiber laser / J. Nilsson, J.D. Minelly, R. Paschotta, A.C. Tropper, D.C. Hanna // Opt. Lett. - 1998. - Vol. 23. - P. 355-357.

11. Koponen J., Soderlund M., Tammela S., Po H. Photodarkening in ytterbium-doped silica fibers // Proc. SPIE. - 2005. - Vol. 5990, 599008.

12. Photodarkening of alumosilicate and phosphosilicate Yb-doped fibers / A. Shubin, M. Yashkov, M. Melkumov, S. Smirnov, I. Bufetov, E. Dianov // Proc. The European Conference on Lasers and Electro-Optics (CLEO_E). - Munich, Germany, 2007. - CJ3_1.

13. Photodarkening and photobleaching of an ytterbium-doped silica double-clad LMA fiber / I. Manek-Hönninger, J. Boullet, T. Cardinal, F. Guillen, S. Ermeneux, M. Podgorski, R. Bello Doua, F. Salin // Opt. Express - 2007. - Vol. 15. - P. 1606-1611. 
14. Gamma-radiation-induced photodarkening in ytterbium-doped silica glasses / T. Arai, K. Ichii, S. Tanigawa, M. Fujimakia // Proc. SPIE 7914, Fiber Lasers VIII: Technology, Systems, and Applications. - 2011. 79140K. DOI:10.1117/12.879800

15. Luminescence and photoinduced absorption in ytterbium-doped optical fibres / A.A. Rybaltovsky, S.S. Aleshkina, M.E. Likhachev, M.M. Bubnov, A.A. Umnikov, M.V. Yashkov, A.N. Gur'yanov, E.M. Dianov // Quantum Electronics. - 2011. - Vol. 41. - P. 1073-1079.

16. Роль кислородно-дырочных центров окраски в механизме фотопотемнения фосфоросиликатных световодов, легированных оксидом иттербия / А.А. Рыбалтовский, А.А. Умников, К.К. Бобков, Д.С. Липатов, А.Н. Романов, М.Е.Лихачев, В.Б.Сулимов, А.Н. Гурьянов, М.М. Бубнов, Е.М. Дианов // Квантовая электроника. - 2013. - № 43. C. $1037-1042$.

17. Возбуждение состояния с переносом заряда как основной механизм фотопотемнения алюмосиликатных световодов, легированных оксидом иттербия / К.К. Бобков, А.А. Рыбалтовский, В.В. Вельмискин, М.Е. Лихачев, М.М. Бубнов, Е.М. Дианов, А.А. Умников, А.Н. Гурьянов, Н.Н. Вечканов, И.А. Шестакова // Квантовая электроника. - 2014. № 44. - С. 1129-1135.

18. Degradation-resistant lasing at $980 \mathrm{~nm}$ in a $\mathrm{Yb} / \mathrm{Ce} / \mathrm{Al}$-doped silica fiber / P. Jelger, M. Engholm, L. Norin, F. Laurell // J. Opt. Soc. Am. B. 2010. - Vol. 27. - P. 338-342.

19. Effect of the $\mathrm{AlPO}_{4}$ join on the pump-to-signal conversion efficiency in heavily Er-doped fibers / M.E. Likhachev, M.M. Bubnov, K.V. Zotov, D.S. Lipatov, M.V. Yashkov, A.N. Guryanov // Opt. Lett. - 2009. - Vol. 34. P. 3355-3357.

20. Influence of aluminum-phosphorus codoping on optical properties of ytterbium-doped laser fibers / S. Unger, A. Schwuchow, S. Jetschke, V. Reichel, M. Leich, A. Scheffel, J. Kirchhof // Proc. SPIE 7212, 72121B1. - 2009.

21. Large-Mode-Area Highly Yb-doped Photodarkening-Free Al2O3P2O5-SiO2-Based Fiber / M. Likhachev, S. Aleshkina, A. Shubin, M. Bubnov, E. Dianov, D. Lipatov, A. Guryanov // CLEO/Europe and EQEC 2011 Conference Digest, OSA Technical Digest (CD) (Optical Society of America). - 2011. - Paper CJ_P24.

22. Фосфороалюмосиликатные световоды, легированные оксидом эрбия / М.Е. Лихачев, М.М. Бубнов, К.В. Зотов, О.И. Медведков, Д.С. Ли- 
патов, М.В. Яшков, А.Н. Гурьянов // Квантовая электроника. - 2010. № 40. - С. 633-638.

23. Study on the output properties of fiber lasers operating near $980 \mathrm{~nm} /$ Liu Ying, Cao Jianqiu, Xiao Hu, Guo Shaofeng, Si Lei, Huang Liangjin // J. Opt. Soc. Am. B. - 2013. - Vol. 30. - P. 266-274.

\section{References}

1. Bartolacci C., Laroche M., and Gilles H. All-fiber Yb-doped CW and pulsed laser sources operating near $980 \mathrm{~nm}$. Advanced Solid-State Photonics, OSA Technical Digest (CD) (Optical Society of America), 2011, paper ATuB9.

2. Fu L.B., Ibsen M., Ridcharson D.J., Payne D.N. $977 \mathrm{~nm}$ all fiber DFB laser. IEEE Phot. Tech.Let., 2004, vol. 16, pp. 2442-2445.

3. Zenteno L. A., Minelly J. D., Dejneka M., Crigler S. 0.65 W singlemode $\mathrm{Yb}$-fiber laser at $980 \mathrm{~nm}$ pumped by $1.1 \mathrm{~W}$ Nd:YAG. Advanced Solid State Lasers, OSA Technical Digest Series (Optical Society of America), 2000, paper TuC8.

4. Kurkov A.S., Medvedkov O.I., Paramonov V.M., Vasiliev S.A., Dianov E.M., Solodovnikov V. High-power Yb-doped double-clad fiber lasers for a range of 0.98-1.04 $\mu \mathrm{m}$. Optical Amplifiers and Their Applications, OSA Technical Digest Series (Optical Society of America), 2001, paper OWC2.

5. Ylä-Jarkko K.H., Selvas R., Son D.B.S., Sahu J.K., Codemard C.A., Nilsson J., Alam S.A., Grudinin A.B. A 3.5 W 977 nm Cladding-pumped Jacketed Air-Clad Ytterbium-Doped Fiber Laser. Advanced Solid-State Photonics, vol. 83 of OSA Trends in Optics and Photonics (Optical Society of America), 2003, paper 103.

6. Leich M., Jäger M., Grimm S., Dellith J. [et al.] Investigation of a large core $976 \mathrm{~nm} \mathrm{Yb}$ fiber laser for high brightness fiber-based pump sources. Proc. SPIE 8961, Fiber Lasers XI: Technology, Systems, and Applications, 2014, 89610Z. DOI: 10.1117/12.2039169

7. Kurkov A.S., Dianov E.M., Paramonov V.M., Medvedkov O.I., Vasiliev S.A., Solodovnikov V., Zhilin V., Laptev A.Y., Umnikov A.A., Guryanov A.N. Efficient $\mathrm{Yb}$ fiber laser at $980 \mathrm{~nm}$ pumped by the high-brightness semiconductor source. Conference on Lasers and Electro-Optics, OSA Technical Digest (Optical Society of America), 2001, paper CTuQ2. 8. Soh D.B.S., Codemard C., Sahu J.K., Nilsson J., Philippov V., Alegria C., 
Jeong Y. A $4.3 \mathrm{~W} 977 \mathrm{~nm}$ ytterbium-doped jacketed-air-clad fiber amplifier. Advanced Solid-State Photonics, OSA Technical Digest (Optical Society of America), 2004, paper MA3.

9. Boullet J., Zaouter Y., Desmarchelier R., Cazaux M., Salin F., Saby J., Bello-Doua R., and Cormier E. High power ytterbium-doped rod-type threelevel photonic crystal fiber laser. Opt. Express, 2008, vol. 16, pp. 17891-17902.

10. Nilsson J., Minelly J.D., Paschotta R., Tropper A.C., and Hanna D.C. Ring-doped cladding-pumped single-mode three-level fiber laser. Opt. Lett., 1998, vol. 23, pp. 355-357.

11. Koponen J., Soderlund M., Tammela S., Po H. Photodarkening in ytterbium-doped silica fibers. Proc. SPIE, 2005, vol. 5990, 599008.

12. Shubin A., Yashkov M., Melkumov M., Smirnov S., Bufetov I., Dianov E. Photodarkening of alumosilicate and phosphosilicate Yb-doped fibers. Proc. The European Conference on Lasers and Electro-Optics (CLEO_E). Munich, Germany, 2007, CJ3_1.

13. Manek-Hönninger I., Boullet J., Cardinal T., Guillen F., Ermeneux S., Podgorski M., Bello Doua R., Salin F. Photodarkening and photobleaching of an ytterbium-doped silica double-clad LMA fiber. Opt. Express, 2007, vol. 15, pp. 1606-1611.

14. Arai T., Ichii K., Tanigawa S., Fujimakia M. Gamma-radiationinduced photodarkening in ytterbium-doped silica glasses. Proc. SPIE 7914, Fiber Lasers VIII: Technology, Systems, and Applications, 2011, 79140K. DOI: $10.1117 / 12.879800$

15. Rybaltovsky A.A., Aleshkina S.S, Likhachev M.E., Bubnov M.M., Umnikov A.A., Yashkov M.V., Gur'yanov A.N., Dianov E.M. Luminescence and photoinduced absorption in ytterbium-doped optical fibres. Quantum Electronics, 2011, vol. 41, pp. 1073-1079

16. Rybaltovskii A.A., Umnikov A.A., Bobkov K.K., Lipatov D.S., Romanov A.N., Likhachev M.E., Sulimov V.B., Gur'ianov A.N., Bubnov M.M., Dianov E.M. Rol' kislorodno-dyrochnykh tsentrov okraski v mehanizme fotopotemneniia fosforosilikatnykh svetovodov, legirovannykh oksidom itterbiia [Role of oxygen-hole color centers in the mechanism of photopotential phosphorothiolate of the optical fiber is doped with ytterbium oxide]. Kvantovaia elektronika, 2013, no. 43, pp. 1037-1042.

17. Bobkov K.K., Rybaltovskii A.A., Vel'miskin V.V., Likhachev M.E., Bubnov M.M., Dianov E.M., Umnikov A.A., Gur'ianov A.N., Vechkanov N.N., Shestakova I.A. Vozbuzhdenie sostoianiia s perenosom zariada kak osnovnoi 
mekhanizm fotopotemneniia aliumosilikatnykh svetovodov, legirovannykh oksidom itterbiia [The excited state with charge transfer as the primary mechanism photopotential aluminosilicate optical fibers doped with ytterbium oxide]. Kvantovaia elektronika, 2014, no. 44, pp. 1129-1135.

18. Jelger P., Engholm M., Norin L., Laurell F. Degradation-resistant lasing at $980 \mathrm{~nm}$ in a $\mathrm{Yb} / \mathrm{Ce} / \mathrm{Al}$-doped silica fiber. J. Opt. Soc. Am. B, 2010, vol. 27, pp. 338-342.

19. Likhachev M.E., Bubnov M.M., Zotov K.V., Lipatov D.S., Yashkov M.V., Guryanov A.N. Effect of the $\mathrm{AlPO}_{4}$ join on the pump-to-signal conversion efficiency in heavily Er-doped fibers. Opt. Lett., 2009, vol. 34, pp. 3355-3357.

20. Unger S., Schwuchow A., Jetschke S., Reichel V., Leich M., Scheffel A., and Kirchhof J. Influence of aluminum-phosphorus codoping on optical properties of ytterbium-doped laser fibers. Proc. SPIE, 2009, 7212, 72121B1.

21. Likhachev M., Aleshkina S., Shubin A., Bubnov M., Dianov E., Lipatov D., and Guryanov A. Large-Mode-Area Highly Yb-doped Photodarkening-Free Al2O3-P2O5-SiO2-Based Fiber. CLEO/Europe and EQEC 2011 Conference Digest, OSA Technical Digest (CD) (Optical Society of America), 2011paper CJ_P24.

22. Likhachev M. E., Bubnov M.M., Zotov K.V., Medvedkov O.I., Lipatov D.S., Iashkov M.V., Gur'ianov A.N. Fosforoaliumosilikatnye svetovody, legirovannye oksidom jerbii [Phosphoroamidite optical fibers doped with erbium oxide]. Kvantovaia elektronika, 2010, no. 40, pp. 633-638.

23. Liu Ying, Cao Jianqiu, Xiao Hu, Guo Shaofeng, Si Lei, and Huang Liangjin, Study on the output properties of fiber lasers operating near 980 nm. J. Opt. Soc. Am. B, 2013, vol. 30, pp. 266-274.

Получено 9.06.2015 